\title{
Combination therapy for an elderly patient with chromoblastomycosis caused by Fonsecaea monophora: a case report
}

\author{
Yu Shen ${ }^{1 \#}$, Boxuan Jiang ${ }^{2 \#}$, Han Zhang ${ }^{2 \#}$, Jinrong Feng ${ }^{3}$, Hui Hua ${ }^{1}$ \\ ${ }^{1}$ Department of Dermatology, Third Affiliated Hospital of Nantong University, Nantong Third People's Hospital, Nantong, China; ${ }^{2}$ School of \\ Medicine, Nantong University, Nantong, China; ${ }^{3}$ Department of Pathogen Biology, School of Medicine, Nantong University, Nantong, China \\ \#These authors contributed equally to this work. \\ Correspondence to: Jinrong Feng. Department of Pathogen Biology, School of Medicine, Nantong University, Nantong, China. \\ Email: jinrong532@163.com; Hui Hua. Department of Dermatology, Third Affiliated Hospital of Nantong University, Nantong Third People's \\ Hospital, Nantong, China. Email: 214972881@qq.com.
}

\begin{abstract}
We report the first case of combined treatment using oral drugs, thermotherapy, and carbon dioxide fractional laser for an elderly patient with skin chromoblastomycosis caused by Fonsecaea monophora. Chromoblastomycosis is a chronic and refractory granulomatous disease of the skin and subcutaneous tissues caused by a group of dematiaceous fungi, which can cause teratogenesis, disability, and even cancer. One of the subtypes, F. monophora, is not only limited to the skin and subcutaneous tissues but also affects the central nervous system. Therefore, a timely and clear diagnosis, as well as active and effective treatment, are particularly important. This case report presents a 75 -year-old male patient whose left forearm had a plaque with mild pruritus for more than three years. The patient's skin lesions were histopathologically examined, and the fungus on the surface of the scabbed skin was examined by fluorescence microscopy and cultured. The strains obtained by the culture were identified by morphological and molecular biology, and a drug susceptibility test was conducted in vitro. Histopathology revealed hyperkeratosis of the epidermis with pseudoepitheliomatous hyperplasia, chronic granulomatous changes in the dermis, and brown thickwalled sclerotic corpuscles both inside and outside giant cells. Septate hyphae and sclerotic corpuscles could be observed in the fungus on the surface of the scabbed skin by fluorescence staining, and black villous colonies could be observed in vitro. Under the scanning electron microscope, rhinocladiella was the primary sporulation type, and the conidia were oval. Molecular identification results showed that the similarity between its internal transcribed spacer (ITS) sequence and that of F. monophora, a Chinese strain (IFM41705), was the highest, reaching $100 \%$. The results of the drug susceptibility test showed that the minimum inhibitory concentrations of itraconazole and voriconazole were $0.125 \mathrm{mg} / \mathrm{L}$ and $0.06 \mathrm{mg} / \mathrm{L}$, respectively. The patient was given oral itraconazole $0.2 \mathrm{qd}$, combined with local thermotherapy and carbon dioxide fractional laser treatment. After 16 weeks, the microscopic examination of the fungus was negative, showing good efficacy.
\end{abstract}

Keywords: Fonsecaea monophora; chromomycosis; molecular identification; carbon dioxide laser; case report

Submitted Oct 27, 2021. Accepted for publication Dec 24, 2021.

doi: $10.21037 / \mathrm{atm}-21-6119$

View this article at: https://dx.doi.org/10.21037/atm-21-6119

\section{Introduction}

Chromoblastomycosis is a chronic refractory granulomatous disease of the skin and subcutaneous tissue caused by a group of dematiaceous fungi, with the skin lesions generally localized. Pathogenic fungi usually invade the skin via local minor trauma. The disease is most common in tropical and subtropical zones, and very few cases occur in the temperate zone. F. monophora is a fungal pathogen that has attracted attention in recent years, and many cases of chromoblastomycosis caused by it have been reported in 
southern China, but it has not been reported in central Jiangsu. Recently, a case of chromoblastomycosis caused by F. monophora was diagnosed and treated in our department, and the isolated pathogen was studied and reported below. We present the following case in accordance with the CARE reporting checklist (available at https://atm.amegroups. com/article/view/10.21037/atm-21-6119/rc).

\section{Case presentation}

\section{Subject and methods}

\section{Subject}

The patient was a 75-year-old male from Nantong, Jiangsu Province. His left forearm had a plaque with slight itching for more than three years. Three years ago, the patient developed localized mung bean-sized red papules with slight itching after a suspected insect bite on the left forearm, which did not attract attention. Later, the rash slowly expanded. One month ago, the patient came to our hospital due to aggravated skin lesions after topical application of hormone ointment. Over the course of the disease, there was no cough, expectoration, night sweats, or anorexia, and his stools and urination were normal. The patient was previously healthy, and there were no individuals with similar diseases in his family. Laboratory tests showed normal routine blood and urine tests, and normal liver and kidney function tests. Physical examination was unremarkable. Dermatology showed an irregular reddishbrown plaque of about $5 \mathrm{~cm} \times 3.5 \mathrm{~cm}$ on the extensor side of the left forearm, with a clear boundary, high margin, a dark brown punctate scab on the medial side of the bulge, slight atrophy of the center of the lesion, and telangiectasia (Figure 1A). All procedures performed in this study were in accordance with the ethical standards of the institutional and/or national research committee(s) and with the Helsinki Declaration (as revised in 2013). Written informed consent was obtained from the patient for publication of this case report and accompanying images. A copy of the written consent is available for review by the editorial office of this journal.

\section{Methods}

\section{Mycological examination}

(I) Black punctate crusts on the surface of the lesions were removed for fungal fluorescence staining (Nanjing Hanrui Biotechnology Co. Ltd.) and observed under a fluorescence microscope; (II) two additional pieces of black punctate crust on the surface of the lesion were cultured on solid Sabouraud dextrose agar (SDA) in an incubator at 25 and $37^{\circ} \mathrm{C}$.

\section{Histopathological examination}

A piece of tissue from the elevated margin of the lesion was excised and fixed in 10\% formalin and embedded in paraffin for section cutting, followed by hematoxylin-eosin (HE) staining and observation under light microscopy.

\section{Molecular strain identification}

The strain to be tested was streaked on SDA plates. Ricesized fungi were collected, dissolved in $100 \mu \mathrm{L}$ Tris-EDTA (TE) buffer, treated at $100{ }^{\circ} \mathrm{C}$ for $10 \mathrm{~min}$, centrifuged at $10,000 \mathrm{rpm}$ for $10 \mathrm{~min}$, and $2 \mu \mathrm{L}$ of the supernatant (raw DNA extract) was directly used for the PCR reaction. PCR amplification was performed using OneTaq (New England Biolabs, USA) polymerase, with ITS1 and ITS4 as the primers. The PCR reaction conditions were $95^{\circ} \mathrm{C}$ for $5 \mathrm{~min}$; then $95^{\circ} \mathrm{C}$ for $30 \mathrm{~s}, 55^{\circ} \mathrm{C}$ for $30 \mathrm{~s}$, and $72{ }^{\circ} \mathrm{C}$ for $60 \mathrm{~s}$, for a total of 30 cycles; then $72{ }^{\circ} \mathrm{C}$ for $5 \mathrm{~min}$. After completing the PCR reaction, $3 \mu \mathrm{L}$ of the product was subjected to $1.5 \%$ agarose gel electrophoresis, and the remaining product was sent to the General Biological Company for twodimensional sequencing detection using ITS1 and ITS4 primers.

\section{In vitro antimicrobial susceptibility testing}

An ATB Funguskit 14204 (BioMerieux, FRA) was used for the antimicrobial susceptibility test. An appropriate amount of fungus was scraped from the surface of the SDA medium, placed in $5 \mathrm{~mL}$ of sterile water, mixed well in a shaker for $2 \mathrm{~min}$, and then the bulk cells were filtered off using four layers of sterile gauze. The fungal solution concentration was adjusted to OD600 $=0.5$ (approximately $2 \mathrm{McF}$ ). A diluted fungal solution $(20 \mu \mathrm{L})$ was added into the ATB F2 culture medium provided with the kit, thoroughly shaken, and mixed well. The fungal solution $(135 \mu \mathrm{L})$ was placed into each test well according to the kit directions, incubated at $30^{\circ} \mathrm{C}$ for $3-4$ days, and the test results were observed.

\section{Results}

\section{Mycological results}

Fungal fluorescence staining of the crusted skin showed septate hyphae and sclerotic corpuscles of varying lengths (Figure $1 B$ ), and some of the sclerotic corpuscles showed septa (Figure 1C). After culturing the black punctate crust for 5 days, the results showed colonies about $0.3-0.5 \mathrm{~cm}$ in diameter, with a black villous surface and a slightly elevated 

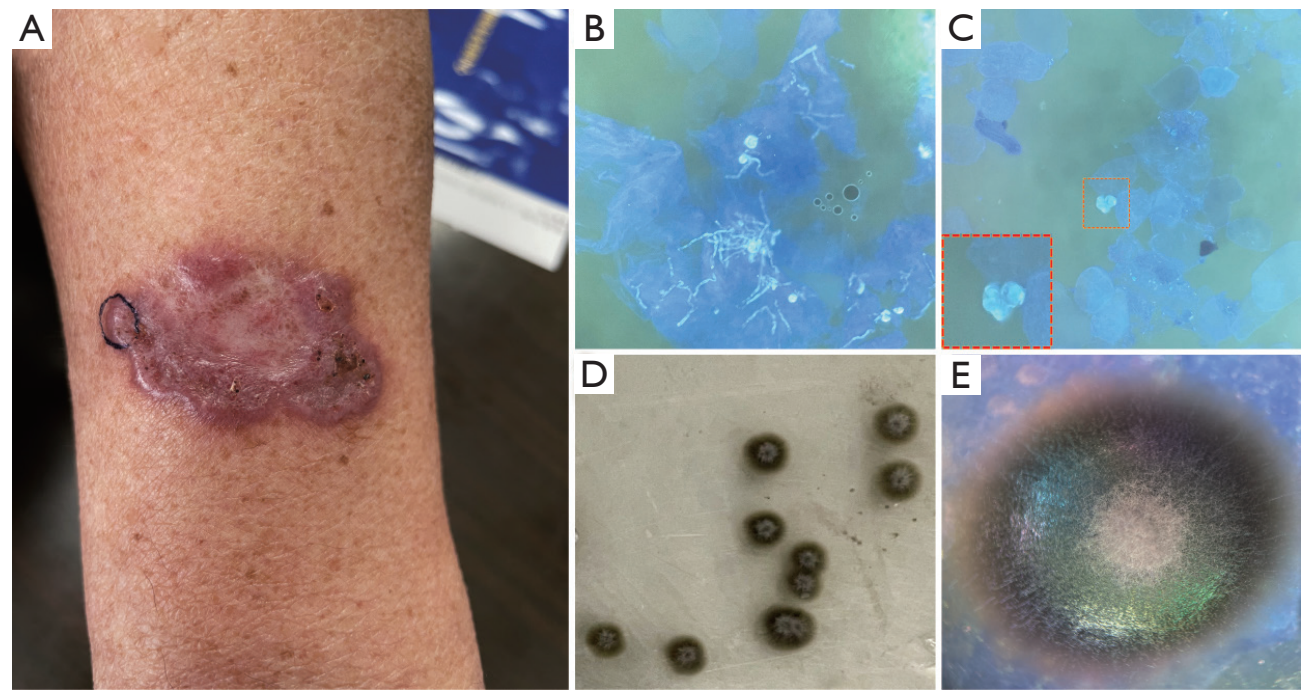

Figure 1 Clinical appearance of chromoblastomycosis lesions in the patient and results of the fungal examination. (A) The initial appearance of the lesion before treatment as an outpatient. (B,C) Fluorescent staining of the skin lesions. A sclerotic body was shown in panel B. (D) The colony morphology on solid SDA media. (E) The colony morphology under a dermoscopy. SDA, sabouraud dextrose agar.

center that was grayish-white (Figure 1D). Through dermoscope, hemispherical colonies were observed, with three distinct demarcation zones; the base was dark brown, the second layer was dense silver-white filaments, and the center was clumped white floccules (Figure 1E). Scanning electron microscopy showed that dense hyphae were predominant in the dark brown site at the periphery of the colony (Figure $2 A$ ), beak cladosporidia were predominant in the center, conidia were oval, and multiple conidia were arranged at the apex of the conidiophores (Figure 2B). The liquid culture of the fungi showed coracoid sporophytic and bottle-type conidiophores (Figure 2C).

\section{Histopathological results}

The histopathological results showed epidermal hyperkeratosis with pseudoepithelial rumen hyperplasia, many types of epithelial cells, multinucleated giant cells in the dermis with microabscess formation, tan thick-walled sclerotic corpuscles inside and outside the giant cells, and septa in some of the sclerotic corpuscles (Figure 3A). Periodic Acid-Schiff (PAS) staining showed reddish-brown thick-walled sclerotic corpuscles in the dermis (Figure 3B).

\section{Strain identification results}

The genomic DNA was extracted, and PCR was performed using the universal fungal primers ITS1 and ITS4, which amplified a significant single band with a size of about $0.6 \mathrm{~kb}$, consistent with the theoretical value (Figure 3C). After sequencing, the PCR product was found to be similar to many strains of the $F$. monophora sequence in the GenBank database (https://www.ncbi.nlm.nih.gov/), with a similarity of more than $99 \%$. Compared with the sequences in the ISHAM Fungal Database (https://its.mycologylab. org/page/Alignment), the PCR product was found to be similar to a Chinese F. monophora strain (IFM41705), with a similarity of $100 \%$. Based on the above results, the pathogen found in this study was identified as F. monophora.

\section{Results of antimicrobial susceptibility testing}

After preparing the fungal solution according to the manufacturer's directions and culturing for the required time, the fungi in the control group grew well. In the drug group, itraconazole and voriconazole had the best antifungal effect, and almost no fungal growth was observed even in the lowest concentration test wells (itraconazole, $0.125 \mathrm{mg} / \mathrm{L}$; voriconazole, $0.06 \mathrm{mg} / \mathrm{L}$ ). 5 -Fluororotic acid had some antifungal ability, and there was weak fungal growth. Fluconazole had a weak antifungal effect, and high concentrations (greater than $16 \mathrm{mg} / \mathrm{L}$ ) were needed to inhibit fungal growth. Amphotericin B had the lowest antifungal effect, and fungal growth was still observed at 

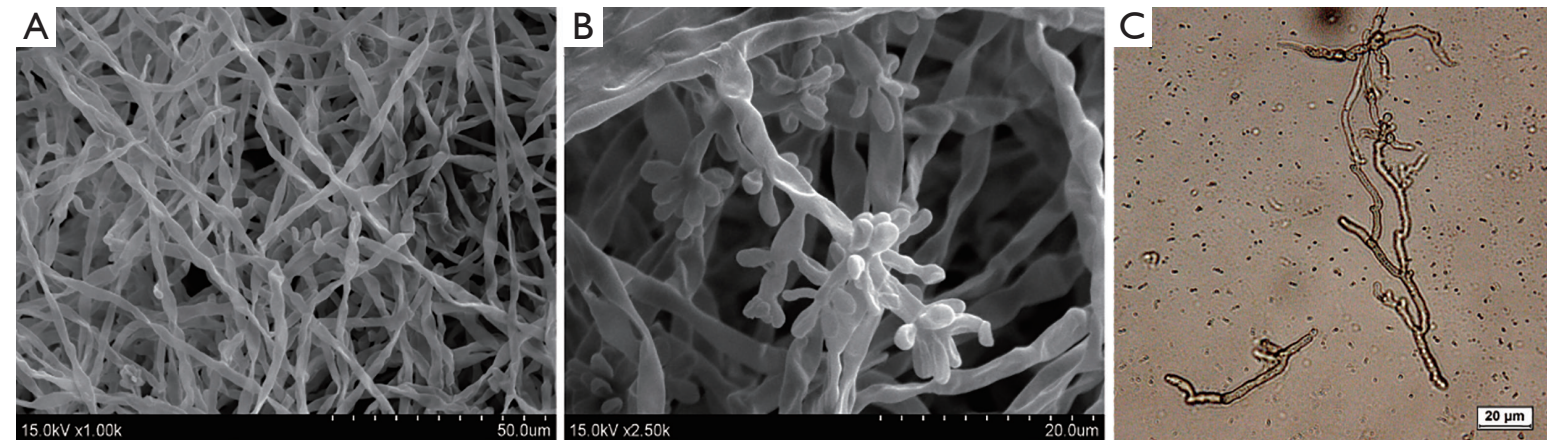

Figure 2 Mycelial morphology. (A,B) SEM results. (C) Liquid culture results. SEM, scanning electron microscope.
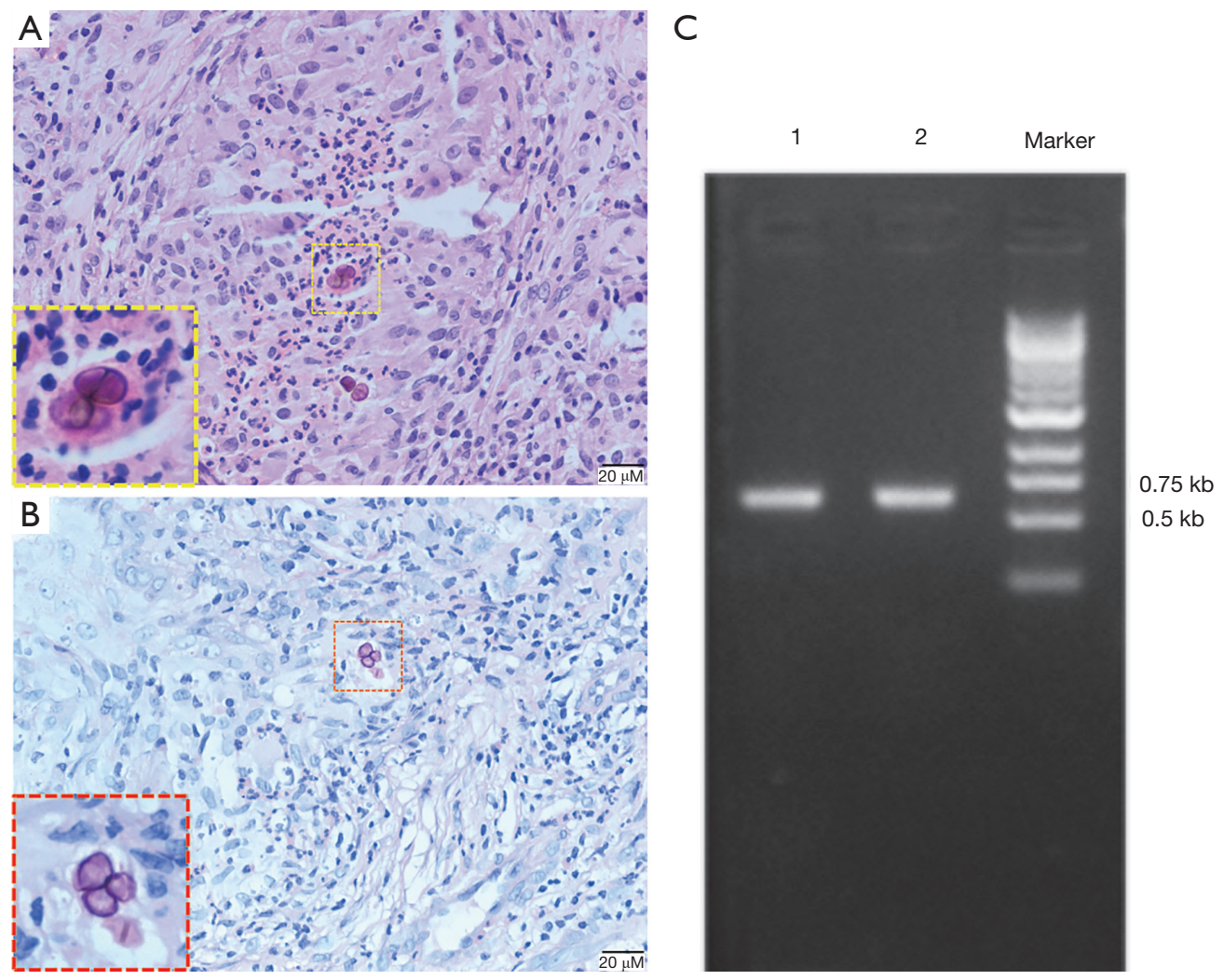

Figure 3 The histopathological examination of skin lesions and molecular identification of strains. (A) HE staining ( $\times 400$ ). (B) PAS staining (×400). (C) PCR amplification results of the fungal ITS sequence. HE, hematoxylin-eosin; PAS, periodic acid-schiff; PCR, polymerase chain reaction; ITS, internal transcribed spacer.

the highest concentration $(16 \mathrm{~g} / \mathrm{L})$. The results indicated that itraconazole and voriconazole had the strongest anti- $F$. monophora effect.

\section{Treatment}

Based on the clinical presentation, mycological, histopathological, and molecular biological examinations, this case was diagnosed as chromoblastomycosis caused by F.monophora. According to the in vitro antimicrobial susceptibility testing results, the patient was given oral itraconazole capsules $0.2 \mathrm{qd}$, with topical bifonazole gel and amorolfine cream applied alternately, along with adjuvant local heat therapy for 20-30 $\mathrm{min} /$ day. The patient returned 

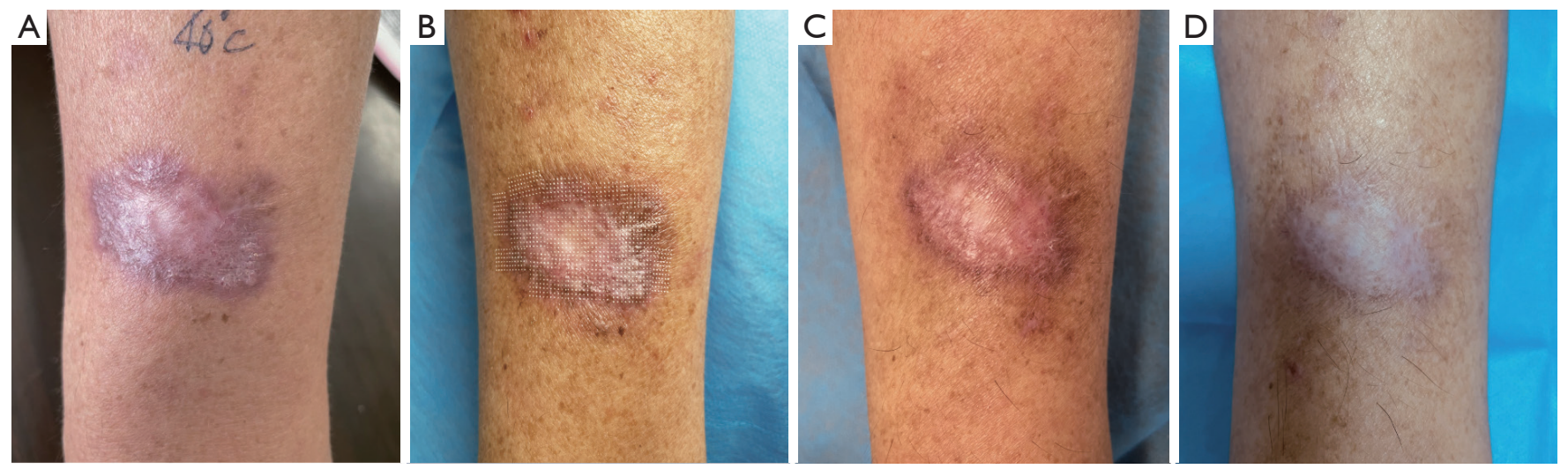

Figure 4 Clinical appearance of chromoblastomycosis lesions after therapy. (A) The lesion after treatment with itraconazole for 8 weeks. (B) The immediate effect on the lesion following the treatment of $\mathrm{CO}_{2}$ fractional photothermolysis. (C) The lesions after 16 weeks of treatment with itraconazole combined with two treatments with $\mathrm{CO}_{2}$ fractional photothermolysis. (D) The lesions after 20 weeks of treatment with itraconazole combined with three treatments with $\mathrm{CO}_{2}$ fractional photothermolysis.

regularly every 4 weeks, and after 8 weeks of treatment, the skin lesions were significantly reduced, and the elevated margin gradually flattened (Figure $4 A$ ). Observable spores were scraped from the skin surface for fungal fluorescence microscopy. The treatment continued as before, assisted with carbon dioxide fractional laser treatment (Figure $4 B$ ) using an energy level of $70 \mathrm{~mJ}$ at the elevated site and $60 \mathrm{~mJ}$ medially, with a coverage rate of $11.1 \%$. Topical amorolfine cream was encapsulated for 1 hour after carbon dioxide fractional laser treatment every 4 weeks. After 16 weeks of treatment, the lesion's periphery was significantly flattened (Figure $4 C$ ), and the fungal fluorescence microscopy was negative.

\section{Discussion}

Chromoblastomycosis has a worldwide distribution and is mainly endemic in hot and humid areas of the tropics and subtropics; males aged 20-60 years appear to be most vulnerable (1). The primary pathogenic fungi causing the disease include Fonsecaea pedrosoi, Phialophra verrucosa, Chladophialophora carrionii, Fonsecaea compacta, F. monophora, and Rhinocladiella acquaspersa (1). Among the reported cases in China, C. carrionii was identified as the predominant pathogen in the north, whereas $F$. monophora was the most likely major pathogen in the south $(2,3)$. By analyzing the clinical data of 20 cases of chromoblastomycosis caused by F.monophora reported in China, we found that cases were mainly distributed in the Guangxi Province and Yungui region (17 cases, $85 \%$ ), and rare in the northern part of
China (2 cases, 10\%). Patients were aged 36-78 years, with a mean age of 59.68 years, and males accounted for $90 \%$ of cases (Table 1). This case was the first report in central Jiangsu Province.

In this case, the patient was a retired worker from Nantong, Jiangsu, who had lived locally for a long time and had no history of travel before the onset of the disease or until the present time. Before the onset of the disease, the patient denied a history of trauma, emphasizing the presence of red papules after insect bites, which gradually and slowly increased. Individual reports of chromoblastomycosis caused by mosquito bites have previously been diagnosed and reported in China (4). F. monophora, a biphasic fungus, is a saprophytic fungal pathogen that mainly parasitizes decomposing plants and soils, such as rotten wood and dead grass. Once they enter the skin tissue through minor skin injuries, chromoblastomyces convert the hyphae from the rotting vegetation into sclerotic corpuscles (5). After extensive questioning of his medical history, the patient revealed that he enjoyed gardening. The authors speculate that chromoblastomyces present in the soil when the patient was gardening might have entered the skin through a slight mosquito bite wound on his forearm. The initial lesion was a single, slightly itchy, red papule at the forearm bite site, which slowly expanded outwards along the periphery and gradually formed plaques and nodules, with verrucous and proliferative changes on the surface and black dot-like crusts.

Chromoblastomycosis has a long and chronic course and can be teratogenic, disabling, and may even become 


\section{Page 6 of 8}

Table 1 The characteristics of 20 cases of chromoblastomycosis caused by F. monophora

\begin{tabular}{lcc}
\hline General condition & Cases $(\mathrm{n})$ & Percent (\%) \\
\hline Gender & 18 & 90 \\
Male & 2 & 10 \\
Female & 9 & \\
Region & 1 & 45 \\
Upper limbs & 10 & 5 \\
Shoulder & & 50 \\
Lower limbs & 10 & 50 \\
Area & 6 & 30 \\
Guangdong & 1 & 5 \\
Guangxi & 2 & 10 \\
Yunnan \& Guizhou & 1 & 5 \\
Hebei & & \\
North Jiangsu & & \\
\hline
\end{tabular}

cancerous (6-8). Therefore, timely and definite diagnosis, as well as active and effective treatment, are required. $F$. monophora is a type of chromoblastomyce that is neurotropic, virulent, and causes chromoblastomycosis and phaeohyphomycosis. It can cause infections in multiple organ systems, including the skin and the brain. Previous studies have reported cases of phaeohyphomycosis caused by $F$. monophora $(9,10)$. Hence, when the isolated strain is identified as chromomycosis, it is essential that further molecular biological testing should be performed to determine whether it is $F$. monophora so as to guide clinical treatment and epidemiology. Ajello [1974] and McGinnis [1983] diagnosed phaeohyphomycosis and distinguished it from chromomycosis based on the parasitic histological morphology of phaeohyphal fungal infections in which yeast-like cells, pseudohyphae, or hyphae-like structures form in tissues (11). In our patient, septated sclerotic corpuscles and septated hyphae of varying lengths were found through microscopic examination of the fungi, tan and red-brown sclerotic corpuscles in the dermis were found histopathologically, but no hyphae were found by PAS staining. A diagnosis of chromoblastomycosis caused by $F$. monophora was made based on the combined clinical manifestations and molecular biological findings. Chromoblastomycosis fungal smears rarely show germinating sclerotic corpuscles and hyphae, but they appeared in this case, which might have been caused by selfapplication of glucocorticoid ointment to inhibit the local immune reaction at the lesion site (12). Taken together, identifying the specific pathogen of chromoblastomycosis may be crucial for treatment.

The treatment of this disease remains a global challenge. Chromoblastomyces pathogens form sclerotic corpuscles in the tissue, which often cause hypertrophic scars or fibrosis, making it difficult for topical drugs to penetrate. This disease has no possibility of healing spontaneously. According to statistics, the condition has a recurrence rate of more than $40 \%$ (13). At present, standard clinical treatments include surgery, physical therapy, chemotherapy, and combination therapy (14). In our case, the patient was treated with combination therapy. He was given oral itraconazole $0.2 \mathrm{qd}$, alternating topical bifonazole gel and amorolfine cream, and local heat therapy, requiring a controlled temperature of $40-42{ }^{\circ} \mathrm{C}$ to avoid hypothermic burns. A previous study demonstrated that strains could not grow at $40{ }^{\circ} \mathrm{C}(15)$. The thermal diffusion effect also promoted the penetration of drugs applied to the surface skin lesions into the deep tissues. Furthermore, the thermal effect increased local blood circulation, facilitated the dissipation of inflammation, and enabled oral antifungal drugs to reach more lesion sites. After 8 weeks of oral medication combined with thermotherapy, the patient's skin lesions shrank significantly, and the high margins gradually flattened. Treatment with oral itraconazole requires a 6-12-month course of treatment (13). Considering the potential development of oral drug side effects in older patients, and after adequate consultation, carbon dioxide fractional laser-assisted transepidermal drug delivery was added at weeks 9 and 13 . After 16 weeks of treatment, the lesion's periphery was significantly flattened, and the fungal microscopy was negative. Carbon dioxide fractional laser treatment can increase the penetration of drugs (16). It can also diffuse into skin lesions and surrounding adjacent tissues through the selective photothermal effect or transmit heat to the surrounding area through optical radiation, producing a thermal effect (17) that is not conducive to the growth of pathogenic fungi. The patient failed to receive timely medical treatment over the long-term course of the disease, and a mild atrophic scar had appeared in the center of the skin lesion, which was an important indicator for carbon dioxide fractional laser treatment (17). While increasing the drug penetration, the atrophic scar tissue was also repaired, which improved the patient's quality of life. 
Table 2 The treatment results of 20 cases of chromoblastomycosis caused by F. monophora

\begin{tabular}{|c|c|c|c|c|c|}
\hline Therapeutic regimen & Cases (n) & Cured (n) & Better (n) & Uncured (n) & Loss (n) \\
\hline \multicolumn{6}{|l|}{ Systematic drug therapy } \\
\hline Itraconazole & 8 & & 8 & & \\
\hline Terbinafine & 3 & 1 & 1 & & 1 \\
\hline Itraconazole + Terbinafine & 5 & 3 & 2 & & \\
\hline \multicolumn{6}{|l|}{$\begin{array}{l}\text { Systematic drugs combined with } \\
\text { physical therapy }\end{array}$} \\
\hline Itraconazole + Thermotherapy & 2 & & 2 & & \\
\hline Terbinafine + Thermotherapy & 1 & & 1 & & \\
\hline \multicolumn{6}{|l|}{ Topical medication } \\
\hline
\end{tabular}

Analysis based on the clinical data of 20 cases of chromoblastomycosis caused by $F$. monophora reported in China showed that 16 patients received at least one oral antifungal drug, mainly oral itraconazole and/or terbinafine, without other combinations; three patients received oral itraconazole or terbinafine combined with hyperthermia, and one patient was treated with the topical compound ketoconazole alone (Table 2). Furthermore, it is worth pointing out that the current patient is an elderly man, which restricts our treatment options. A high level of itraconazole could be considered if it is a young patient, such as $400 \mathrm{mg} / \mathrm{d}$. Alternatively, combination therapy of itraconazole and terbinafine may optimize drug therapy. In general, the treatment in our study is significantly improved and showed satisfying efficacy for this unusual case.

In addition to the classic azole and acrylamide drugs, some other therapies may be considered for this fungal pathogen in the future. 5-ALA PDT has been proven to be useful for treating the infection caused by $F$. monophora both in vivo and in vitro (18). The combined therapy of 5-ALA PDT and antifungal drugs should also have great efficacy. For example, Huang used ALA-PDT to cure a chromoblastomycosis patient with leucopenia, suggesting an adaptable method for curing refractory cases of chromoblastomycosis (19). Furthermore, some immunomodulators, such as glucan or imiquimod, may increase antifungal efficacy. For instance, combined therapy of injection of glucan and oral medication of itraconazole cured a patient infected with chromoblastomycosis, who has received the treatment of itraconazole and terbinafine for 3 years but with no significant efficacy (20).

Our patient is the first case treated with oral drugs, thermotherapy, and carbon dioxide fractional laser in China. The clinical trial has proved that the combined treatment is safe and effective, with good patient satisfaction. This is the first case of chromoblastomycosis diagnosed and treated in our department without previous experience in therapy. At the time of submission, the patient continues to attend regular outpatient follow-up every 4 weeks and the lesion on the arm recovers significantly after 20 weeks (Figure 4D).

\section{Acknowledgments}

Funding: None.

\section{Footnote}

Reporting Checklist: The authors have completed the CARE reporting checklist. Available at https://atm.amegroups. com/article/view/10.21037/atm-21-6119/rc

Conflicts of Interest: All authors have completed the ICMJE uniform disclosure form (available at https://atm. amegroups.com/article/view/10.21037/atm-21-6119/coif). The authors have no conflicts of interest to declare.

Ethical Statement: The authors are accountable for all aspects of the work in ensuring that questions related to the accuracy or integrity of any part of the work are appropriately investigated and resolved. All procedures 
performed in this study were in accordance with the ethical standards of the institutional and/or national research committee(s) and with the Helsinki Declaration (as revised in 2013). Written informed consent was obtained from the patient for publication of this case report and accompanying images. A copy of the written consent is available for review by the editorial office of this journal.

Open Access Statement: This is an Open Access article distributed in accordance with the Creative Commons Attribution-NonCommercial-NoDerivs 4.0 International License (CC BY-NC-ND 4.0), which permits the noncommercial replication and distribution of the article with the strict proviso that no changes or edits are made and the original work is properly cited (including links to both the formal publication through the relevant DOI and the license). See: https://creativecommons.org/ licenses/by-nc-nd/4.0/.

\section{References}

1. Bolognia JL, Schaffer JV, Cerroni L. Dermatology. Zhu Xu, Wang B, Sun J, et al. The 4th Edition. Beijing: Peking University Medical Press, 2019:1495.

2. De Hoog GS, Attili-Angelis D, Vicente VA, et al. Molecular ecology and pathogenic potential of Fonsecaea species. Med Mycol 2004;42:405-16.

3. Xi L, Sun J, Lu C, et al. Molecular diversity of Fonsecaea (Chaetothyriales) causing chromoblastomycosis in southern China. Med Mycol 2009;47:27-33.

4. Sun QL, Yu M, Chen J, et al. Retrospective analysis of chromoblastomycosis in mainland China: a review of 52 cases. Chinese Journal of Mycology 2020;15:101-5.

5. Wu W,Wang JD, Li W, et al. Multiforme Rashes of Chromoblastomycosis Caused by Fonsecaea Monophora. The Chinese Journal of Dermatovenereology 2019;(2):184-7.

6. Zhao B. Chinese Clinical dermatology. The 2nd Edition. Nanjing: Jiangsu Phoenix Science Press, 2017:612.

7. $\mathrm{Hu} \mathrm{BQ}$. Research progress of chromoblastomycosis. Medical Review 2009;15:851-3.

8. Azevedo CM, Marques SG, Santos DW, et al. Squamous cell carcinoma derived from chronic chromoblastomycosis in Brazil. Clin Infect Dis 2015;60:1500-4.

9. Koo S, Klompas M, Marty FM. Fonsecaea monophora cerebral phaeohyphomycosis: case report of successful surgical excision and voriconazole treatment and review.
Med Mycol 2010;48:769-74.

10. Doymaz MZ, Seyithanoglu MF, Hakyemez İ, et al. A case of cerebral phaeohyphomycosis caused by Fonsecaea monophora, a neurotropic dematiaceous fungus, and a review of the literature. Mycoses 2015;58:187-92.

11. Wang DL. Medical mycology - Guidelines for laboratory testing. The 1st Edition. Beijing: People's Medical Publishing House, 2005:304.

12. Li MR, Chen YD, Yin SC, et al. Chromoblastomycosis with unusual polymorphic sclerotic bodies: case study and review of the literature. Chinese Journal of Mycology 2016;11:213-6.

13. James WD, Berger TG, Elston DM. Andrews' Diseases of the Skin. Lei Tiechi et al. The 12th edition. Beijing: Science Press, 2019:311.

14. Shang PP, Zhang FR. Update of chromoblastomycosis treatment. China Journal of Leprosy and Skin Diseases 2017;33:125-8.

15. Liu HF, Xue RZ, Huang JM, et al. Clinical analysis of chromoblastomycosis and identification of Fonsecaea monophora. China Tropical Medicine 2010;10:1062-4.

16. Molu Ozukum. Experimental study on transdermal penetration of topical drugs assisted by ultra-pulsed carbon dioxide lattice laser. Dalian: Dalian Medical University, 2016.

17. Xiang HL, Zhou ZC. Principle and Technology of skin beauty laser therapy. Beijing: People's Medical Publishing House, 2014:62-63.

18. Hu Y, Qi X, Sun H, et al. Photodynamic therapy combined with antifungal drugs against chromoblastomycosis and the effect of ALA-PDT on Fonsecaea in vitro. PLoS Negl Trop Dis 2019;13:e0007849.

19. Huang X, Han K, Wang L, et al. Succesful treatment of chromoblastomycosis using ALA-PDT in a patient with leukopenia. Photodiagnosis and Photodynamic Therapy 2019;26:13-4.

20. Azevedo Cde M, Marques SG, Resende MA, et al. The use of glucan as immunostimulant in the treatment of a severe case of chromoblastomycosis. Mycoses 2008;51:341-4.

Cite this article as: Shen Y, Jiang B, Zhang H, Feng J, Hua H. Combination therapy for an elderly patient with chromoblastomycosis caused by Fonsecaea monophora: a case report. Ann Transl Med 2022;10(2):114. doi: 10.21037/atm-216119 